\title{
Genetic Map Construction and Quantitative Trait Locus (QTL) Detection of Growth-Related Traits in Litopenaeus vannamei for Selective Breeding Applications
}

\author{
Farafidy Andriantahina ${ }^{1}$, Xiaolin Liu ${ }^{1 *}$, Hao Huang ${ }^{2}$ \\ 1 College of Animal Science and Technology, Northwest A\&F University, Shaanxi Key Laboratory of Molecular Biology for Agriculture, Yangling, Shaanxi, \\ People's Republic of China, 2 Hainan Guangtai Ocean Breeding Company Limited, Haikou, People's Republic of China
}

\begin{abstract}
Growth is a priority trait from the point of view of genetic improvement. Molecular markers linked to quantitative trait loci (QTL) have been regarded as useful for marker-assisted selection (MAS) in complex traits as growth. Using an intermediate $\mathrm{F}_{2}$ cross of slow and fast growth parents, a genetic linkage map of Pacific whiteleg shrimp, Litopenaeus vannamei, based on amplified fragment length polymorphisms (AFLP) and simple sequence repeats (SSR) markers was constructed. Meanwhile, QTL analysis was performed for growth-related traits. The linkage map consisted of 451 marker loci (429 AFLPs and 22 SSRs) which formed 49 linkage groups with an average marker space of $7.6 \mathrm{cM}$; they spanned a total length of $3627.6 \mathrm{cM}$, covering $79.50 \%$ of estimated genome size. 14 QTLs were identified for growth-related traits, including three QTLs for body weight (BW), total length (TL) and partial carapace length (PCL), two QTLs for body length (BL), one QTL for first abdominal segment depth (FASD), third abdominal segment depth (TASD) and first abdominal segment width (FASW), which explained 2.62 to $61.42 \%$ of phenotypic variation. Moreover, comparison of linkage maps between L. vannamei and Penaeus japonicus was applied, providing a new insight into the genetic base of QTL affecting the growth-related traits. The new results will be useful for conducting MAS breeding schemes in L. vannamei.
\end{abstract}

Citation: Andriantahina F, Liu X, Huang H (2013) Genetic Map Construction and Quantitative Trait Locus (QTL) Detection of Growth-Related Traits in Litopenaeus vannamei for Selective Breeding Applications. PLoS ONE 8(9): e75206. doi:10.1371/journal.pone.0075206

Editor: Zhanjiang Liu, Auburn University, United States of America

Received May 21, 2013; Accepted August 10, 2013; Published September 25, 2013

Copyright: () 2013 ANDRIANTAHINA et al. This is an open-access article distributed under the terms of the Creative Commons Attribution License, which permits unrestricted use, distribution, and reproduction in any medium, provided the original author and source are credited.

Funding: This research work was supported by the collaborative project of Northwest A\&F University, China (XNY2013-4), National Ministry of Agricultural Science and Technology, China (2012GB2E200361), and National Fundamental Research Program, China (2006AA10A406). The funders had no role in study design, data collection and analysis, decision to publish, or preparation of the manuscript.

Competing interests: The authors have following competing interests. The author Hao Huang is employed by a commercial company "Hainan Guangtai Ocean Breeding Company Limited”. This does not alter the authors' adherence to all the PLOS ONE policies on sharing data and materials.

*E-mail: liuxiaolin@nwsuaf.edu.cn

\section{Introduction}

Increased market demands for penaeid aquaculture production has determined the progress of genetic improvement approaches focused on both higher productivity and reduction of rearing costs [1]. A major challenge in current biology is to understand the genetic basis of variation for quantitative traits [2]. Quantitative traits are determined by the combined forces of genetic and environmental factors and also the interaction between them [3]. Understanding the relationship between variations in DNA sequences and variations in phenotypes for these quantitative traits will yield insights that are important for enhancing the speed of selective breeding schemes in agriculturally important plants and animals and for predicting adaptive evolution [2].

Litopenaeus vannamei (Pacific whiteleg shrimp) is one of the most important shrimp species farmed worldwide with important values in aquaculture [4]. L. vannamei is also one of the shrimp species with the most extensive selective breeding practices in the world. However, its quantitative trait loci (QTL) associated to growth-related traits has been rarely identified. Growth-related traits (for instance, body weight and body sizes), which are economically important complex traits, have been studied in the genetic improvement researches $[5,6]$. Understanding the genetic basis of these complex traits is important to better conduct the genetic improvement schemes. The most direct technique to identify these loci is by finemapping QTL and identifying positional candidate genes, and to conduct maker-assisted selection (MAS) or gene-assisted selection (GAS). To date, genetic mapping of penaeid species has been slow and concentrated mostly on a few important cultured species such as Penaeus monodon [7], $P$. japonicus [8], L. vannamei [9], and $P$. chinensis [10] using primarily dominant amplified fragment length polymorphism (AFLP) markers and a few codominant simple sequence repeats (SSR) or microsatellite markers. However, no precise QTL associated 
to growth-related traits have been identified based on $L$. vannamei linkage maps. Conducting genetic map construction and QTL analysis are very useful in improving the speed of genetic improvement and employing MAS in L. vannamei.

AFLP markers were reported to show a greater level of polymorphism and informativeness than any other markers [11], and have been widely used in linkage mapping of several shrimp species [7-10]. SSR markers have been increasingly used in the construction of linkage maps in the past decade for aquatic species [10,12-14]. In this research, we constructed a genetic linkage map of $L$. vannamei with AFLP and SSR markers using an intermediate $F_{2}$ cross of slow and fast growth parents and identified 14 QTLs associated with growth-related traits in $L$. vannamei. With the object of gaining new understanding of genes related to growth traits in a commercial penaeid species, the specific objectives of this present research were to determine the loci that are responsible for variation in growth-related quantitative traits and to provide insight into the genetic architecture of the traits that are interesting for breeding applications in L. vannamei.

\section{Materials and Methods}

\section{Animal materials}

The parent stocks of $L$. vannamei used in this research were obtained from a commercial pond of Hainan Guangtai Ocean Breeding Company Limited (Wenchang Breeding Base), China. Following spawning and larval culture, approximately 20,000 progeny were stocked into a $666.6 \mathrm{~m}^{2}(33.33 \mathrm{~m} \times 20 \mathrm{~m} \times 1.5$ $\mathrm{m}$ ) pond at an initial density of 30 per $\mathrm{m}^{2}$ for commercial growout. Animals were harvested after about 6 months of pond-growth. At the harvest, broodstock were selected to establish the laboratory-cultured lines for our research. During harvest, a sample of shrimp was measured for determining the size distribution of pond stocks and weight threshold for selection. From these shrimp, five males and 25 females were chosen as founder parents of slow-growth (SG) line; the same number of shrimp was used to initiate fast-growth (FG) line $A$ further $20 \mathrm{FG}$ and $S G$ males and $20 \mathrm{FG}$ and $S G$ females were also sampled to make crosses between FG and SG parents. After selection, the shrimp were transferred to an experimental laboratory. They were measured for total length (TL), body length (BL), first abdominal segment depth (FASD), third abdominal segment depth (TASD), first abdominal segment width (FASW) and partial carapace length (PCL), using a digital camera and Photoshop software [5]. Shrimp body weights (BW) were determined using an electronic balance. An intermediate $F_{2}$ full-sib population of 43 shrimp, produced by reciprocally mating $4 \mathrm{FG}$ and SG grandparents randomly selected from the above populations and one $F_{1}$ FS maternal and one $F_{1}$ SF paternal parent, were used for map construction. Seven growth-related phenotype traits that were measured according to Andriantahina et al. [5] including BW, TL, BL, FASD, TASD, FASW and PCL, were measured for QTLs identification.

\section{AFLP analysis}

DNA was isolated from the abdominal muscles of $L$. vannamei using a standard phenol-chloroform method [15]. AFLP analysis was performed according to the protocols described by Vos et al. [16] with small modification. Digestionligation reaction mixture contained $3 \mu \mathrm{l}$ genomic DNA (about $200 \mathrm{ng}), 1.1 \mu \mathrm{l} 10 \mathrm{x}$ T4 DNA ligase buffer with ATP, $1.1 \mu \mathrm{l} 0.5$ $\mathrm{M} \mathrm{NaCl}, 0.55 \mu \mathrm{l}$ of $1 \mathrm{mg} / \mathrm{ml}$ bovine serum albumin (BSA), $1 \mathrm{U}$ Msel, $5 \cup$ EcoRI, $1.0 \cup$ T4 DNA ligase, 50 pmol Msel adaptor, $5 \mathrm{pmol} E c o R I$ adaptor and water to bring the final volume to 11 $\mu \mathrm{l}$. The reaction mixture was incubated at $37{ }^{\circ} \mathrm{C}$ for $2 \mathrm{~h}$ and then diluted with $150 \mu \mathrm{TE}_{0.1}(20 \mathrm{mM}$ Tris- $\mathrm{HCl}, 0.1 \mathrm{mM}$ EDTA, $\mathrm{pH}$ 8.0). Preselective amplification was carried out using primers complementary to adaptor sequence without any additional nucleotides at 3 ' end. Selective primers consisted of preselective primers with three selective nucleotides added to 3' end. EcoRI-selective primers were fluorescently 5' labeled with FAM. PCR reactions were performed in a PTC-100 or PTC-200 thermal cycler (MJ Research). PCR products labeled with FAM dye were analyzed with an ABI Prism 310 sequencer.

AFLP markers were named according to the primer pairs used to generate them and also their size. EcoRI- and Mselselective primers were respectively coded by letters and numbers followed by a letter $f$ (fragment) and digits representing the size in base pairs [17]. For instance, D8f100 refers to 100 -bp fragment generated by EcoRI primer D (ACG) and Msel primer 8 (CAT).

\section{Microsatellite genotyping}

Microsatellites were scored using $0.2 \mu \mathrm{M}$ dye-labeled (5FAM) forward primers and $0.5 \mu \mathrm{M}$ reverse primers. PCR reactions were conducted as follows: initial denaturation for 5 min at $94{ }^{\circ} \mathrm{C}$, followed by 35 cycles for $30 \mathrm{~s}$ at $94{ }^{\circ} \mathrm{C}, 30 \mathrm{~s}$ at optimal annealing temperature, and $30 \mathrm{~s}$ at $72{ }^{\circ} \mathrm{C}$, last followed by $10 \mathrm{~min}$ at $72^{\circ} \mathrm{C}$. PCR products labeled with FAM dye were also analyzed with an $A B I$ Prism 310 sequencer.

We tested 147 microsatellite loci recently described by Meehan et al. [18] and Ball et al. [19] for this research work, of which 25 SSR markers were informative. Because most of the original name is too long to be permitted when using mapping software MapMaker/Exp v. 3.0, SSR markers were renamed according to information of the species, trial order in our analysis, and the size of the microsatellite bands. The first letter of marker indicates the species (e.g., v for L. vannamei), followed by digits representing analyzing order in our work and the size of microsatellite band similar with AFLP markers. For example, SSR marker v1f148 indicates that SSR marker of $L$. vannamei was analyzed first in our trials with a size of $148 \mathrm{bp}$.

\section{Linkage analysis and genome coverage}

Genotype data was recorded in a TXT file and imported to Mapmanager QTXb20 software [20]. A Chi-squared test was used to assess Mendelian segregation distortion of all polymorphic loci data in $\mathrm{F}_{2}$ population before linkage analysis. The data set was designated as "intercross" and linkage groups were assigned with $P$-value of $1.0 \mathrm{E}-4$ by using the "Make linkage groups" command and then linkage groups were 
accordingly adjusted by the "Distribute" and "Ripple" command. The consensus linkage map from AFLP and SSR markers was graphically represented using MAPCHART2.1 program [21] based on calculated map distances between markers.

Two approaches were used to assess genome length according to Liu et al. [10]. Under $\mathrm{G}_{\mathrm{e} 1}$ the genome length was assessed by adding $2 \mathrm{~s}$ (where $\mathrm{s}$ is average space of linkage map) to the length of each group, which accounts for chromosome ends. Under $\mathrm{G}_{\mathrm{e}}$, the genome length was estimated by multiplying the length of each linkage group (LG) by $(m+1) /(m-1)$, where $m$ is the number of markers in each LG. The estimated map length is the sum of revised lengths of all LGs [22]. The average of these two estimates was used as estimated genome length $\left(G_{e}\right)$. The observed map length was calculated as total length of map $\left(\mathrm{G}_{\mathrm{of}}\right)$ excluding triplets and doublets (LGs with three and two markers, respectively) or as total length of map including the triplets and doublets $\left(G_{o a}\right)$. The observed genome coverage, $\mathrm{C}_{\text {of }}$ and $\mathrm{C}_{\mathrm{oa}}$, was calculated as $\mathrm{G}_{\mathrm{o}} / \mathrm{G}_{\mathrm{e}}$ and $\mathrm{G}_{\mathrm{oa}} / \mathrm{G}_{\mathrm{e}}$ respectively [23].

\section{QTL analysis}

First, the normality of growth-related quantitative data was estimated using a one-sample Kolmogorov-Smirnov test implemented in SPSS 17.0 package, no deviations from a standard normal distribution were found for the studied traits. QTL analysis was performed by means of composite interval mapping (CIM) [24] implemented in Windows QTL Cartographer 2.5 software [13] using 1000 permutations with significance as 0.05 . A minimum limit of detection (LOD) threshold of 3.0 was used for considering a significant QTL, and the percentage of phenotypic variance explained by each QTL was assessed using Windows QTL Cartographer 2.5.

\section{Comparative mapping}

Comparative genomics can provide valuable information about the architecture and functional organization of a species' genome [25]. A total of 25 AFLP loci placed on the linkage map were used to perform comparative mapping analysis with $P$. japonicus, as the flanking regions of AFLP markers have the ability to identify chromosomal regions that are homologous across species $[11,26]$. The total genome of $P$. japonicus has been sequenced and is closely related to shrimp on phylogenetics. The flanking sequences of $25 \mathrm{~L}$. vannamei AFLP loci were used as queries to blast against the $P$. japonicus whole genome data through National Center of Biotechnology Information (NCBI) (http://www.ncbi.nlm.nih.gov/ genome/).

\section{Results}

\section{Polymorphism and segregation of molecular markers}

A total of 100 AFLP primer combinations in $F_{2}$ population produced approximately 3158 AFLP bands. On average, each primer combination produced 40 to 80 bands with a size between 10 and $1200 \mathrm{bp}$. Among the 3158 bands obtained, 600 were polymorphic accounting for $19 \%$ of the total (Table 1). An average of eight polymorphic markers was detected per primer. Variation was evident in the number of polymorphic fragments revealed by different primer combinations. The level of polymorphism produced by different primer combinations varied considerably from $2 \%$ to $20 \%$. Of the SSR markers genotyped, 25 markers were informative in mapping population of $L$. vannamei and available for map construction (Table 2).

Of the 600 polymorphic AFLP markers, 98 were segregated, of which $31(32 \%)$ deviated from the expected Mendelian 3:1 ratios $(P<0.05)$. Of all the segregating markers (including SSR markers), 513 fitted in the expected segregation ratio and 47 markers showed a significant deviation from the $1: 1$ ratio $(P<$ $0.05)$. Finally, $451(87.91 \%)$ markers were grouped on the linkage map, leaving 63 (12.09\%) markers unlinked.

\section{Linkage mapping}

A total of 600 AFLP markers and 25 SSR markers were employed to construct the linkage map. A total of 451 (34 distored markers) segregating markers were assigned to the growth linkage map (Figure 1). For linkage map, 451 (429 AFLPs, 22 SSRs) were assigned to 49 LGs (more than three markers), which covered $3313.9 \mathrm{cM}\left(\mathrm{G}_{\mathrm{of}}\right)$, in length with an average interval of $7.6 \mathrm{cM}$ and a maximum interval of $32.8 \mathrm{cM}$. Length of LGs ranged from $13.1 \mathrm{cM}$ (LG46) to $141.4 \mathrm{cM}$ (LG1), and the number of markers per group varied from three to 28 with a mean of 8 (Table 3 , Figure 1).

\section{Genome estimation and map coverage}

Assessed genome lengths through the two methods were $4543.0 \mathrm{cM}$ and $4583.0 \mathrm{cM}$ with an average of $4563.0 \mathrm{cM}$. Based on the observed length of the framework map (3313.9 $\mathrm{cM}$ ) and the assessed genome length (4563.0 cM), the framework map had coverage of $72.63 \%$. Map coverage increased to $79.50 \%$ if the doublets were included (Table 3 ).

\section{QTL analysis}

The profiles and characteristics of QTLs associated with seven traits are provided in Table 4, Figure 2. A total of 14 significant QTLs were detected on 9 LGs. Three QTLs (BW-1 to BW-3) were identified for body weight on three different LGs, each of these QTLs explained 2.62 to $18.34 \%$ of phenotypic variation individually. Three QTLs (TL-1 to TL-3) were identified for total length. Two QTLs (BL-1 and BL-2), accounting for $42.78-45.95 \%$ of phenotypic variation, were identified for body length. One QTL was identified for FASD, TASD and FASW, which respectively explained $61.42,56.10$ and $56.20 \%$ of phenotypic variation. Interestingly, three QTLs (PCL-1 to PCL-3) were detected for partial carapace length and accounted for 4.32 to $10.57 \%$ of phenotypic variation.

\section{Comparative mapping}

The flanking sequences of AFLP loci placed on the map were used to compare with genomic DNA sequences of $P$. japonicus. Eight of which were significantly conserved between $L$. vannamei and $P$. japonicus ( $E$-value $\left.<1.0 \times 10^{-4}\right)$. The eight hits were distributed on eight of the 43 chromosomes in $P$. japonicus (Table 5). Unique correspondences were detected in four chromosome pairs (LG15- Chrom9, LG19- Chrom11, 
Table 1. Numbers of polymorphic markers generated by $100 \mathrm{Msel}$ and EcoRI AFLP primer combinations.

\begin{tabular}{|c|c|c|c|c|c|c|c|c|c|c|c|c|c|c|c|c|}
\hline \multirow[t]{2}{*}{ Msel } & \multicolumn{16}{|l|}{ EcoRI } \\
\hline & AAG(A) & ACA(B) & $\mathrm{ACA}(\mathrm{C})$ & ACG(D) & $\operatorname{AGC}(E)$ & AGG(F) & CCG(G) & CCT(H) & CTC(I) & $\operatorname{TAC}(\mathrm{J})$ & ACG(K) & ATA(L) & AAA(M) & $\operatorname{ACC}(N)$ & $\operatorname{AGG(O)}$ & Total \\
\hline ACA:1 & & & 4 & & 1 & 4 & & 1 & 4 & 2 & 1 & 3 & 3 & 1 & 3 & 27 \\
\hline ACC:2 & & 2 & & & 3 & 3 & & & & 3 & 2 & 1 & 3 & 2 & 4 & 23 \\
\hline АСТ:3 & & 3 & 4 & 1 & 3 & 1 & & & 3 & & 1 & 2 & 2 & 1 & & 21 \\
\hline ATC:4 & 5 & & 4 & & 1 & 1 & & & 3 & & & 2 & 2 & 1 & 3 & 22 \\
\hline CAA:5 & 6 & & 3 & 2 & 3 & 4 & 3 & & 2 & 4 & & 1 & 3 & 1 & 2 & 34 \\
\hline CAC:6 & 5 & & & & 4 & 1 & 2 & & & 3 & & 2 & 1 & 1 & 2 & 21 \\
\hline CAG:7 & 6 & 5 & 3 & 2 & 3 & 3 & 2 & 4 & 2 & & 1 & 2 & 3 & 1 & & 37 \\
\hline САТ:8 & 6 & 3 & 4 & 4 & 3 & 3 & 3 & 1 & 3 & 3 & 2 & 1 & & 1 & 2 & 39 \\
\hline CCA:9 & 6 & 1 & 3 & 3 & 1 & 4 & 1 & 3 & 4 & 1 & 3 & 2 & 2 & 1 & & 35 \\
\hline CCG:10 & 3 & 4 & 4 & 3 & 4 & 1 & 3 & 1 & 2 & & 2 & 1 & 3 & 1 & 3 & 35 \\
\hline ССТ:11 & 3 & 1 & 3 & 1 & 1 & 1 & 1 & 2 & 3 & & 1 & 1 & 1 & 1 & & 20 \\
\hline CGA:12 & & 1 & 3 & 1 & & & 1 & 2 & 3 & & 3 & 2 & 3 & 1 & 3 & 23 \\
\hline CGC:13 & & 3 & 1 & 3 & & 2 & 1 & 1 & 3 & & 1 & 1 & & 1 & 1 & 18 \\
\hline CGG:14 & & 1 & & 2 & & 4 & 1 & 1 & & & 1 & 2 & & 1 & 2 & 15 \\
\hline CGT:15 & & 3 & & 1 & 4 & 4 & 1 & 4 & & & 1 & 2 & 2 & 1 & 1 & 24 \\
\hline CTA:16 & 1 & 1 & 2 & 3 & 5 & & 2 & 1 & 3 & 3 & 1 & 2 & 2 & 1 & 1 & 28 \\
\hline CTC:17 & 2 & 1 & 4 & 4 & 4 & 5 & 3 & 3 & 3 & 3 & 1 & 1 & 2 & 1 & & 37 \\
\hline CTG:18 & & 1 & 2 & 4 & & & 2 & 4 & 3 & & 1 & 2 & 3 & 1 & 3 & 26 \\
\hline СТТ:19 & 4 & & 2 & 1 & & 4 & 3 & 5 & 2 & 5 & 1 & 3 & 2 & 1 & & 33 \\
\hline GAA:20 & & & & 1 & & & & 1 & & 4 & 1 & 1 & 2 & 1 & 3 & 14 \\
\hline GAC:21 & 1 & & & & & & & 1 & & & 1 & 2 & 3 & 1 & & 9 \\
\hline GCC:22 & 1 & & 1 & & & & & 1 & 2 & 3 & 1 & 2 & & 1 & 1 & 13 \\
\hline GTA:23 & & & 2 & & & & & & 3 & 3 & 2 & 1 & & 1 & 1 & 13 \\
\hline TAA:24 & & & & & & & & & & & 1 & & 2 & 1 & 2 & 6 \\
\hline TAC:25 & 2 & & & & & & & & & & 2 & & & & 2 & 6 \\
\hline TAG:26 & 5 & & & & & & & & & 2 & 1 & & & & & 8 \\
\hline TCA:27 & 3 & & & & & & & & & 4 & 1 & & & & & 8 \\
\hline TGA:28 & 2 & & & & & & & & & & 1 & & 2 & & & 5 \\
\hline Total & 61 & 30 & 49 & 36 & 40 & 45 & 29 & 36 & 48 & 43 & 34 & 39 & 46 & 25 & 39 & 600 \\
\hline
\end{tabular}

LG31- Chrom23, and LG35- Chrom19). Two AFLP loci (D8f520, M4f100) mapped on LG1 of $L$. vannamei at the locus of $45.9 \mathrm{cM}$ and $60.7 \mathrm{cM}$ were conserved in chromosome 1 and 17 of $P$. japonicus, respectively. Two AFLP loci $(\mathrm{H} 13 f 830$, J3f178) mapped on LG3 and LG25 in this research were both homologous with chromosome 25 of $P$. japonicus.

\section{Discussion}

\section{Polymorphism and segregation of molecular markers}

We constructed a genetic linkage map of $L$. vannamei with AFLP and SSR markers using a full-sib $F_{2}$ intercross design. The relatively high yield of information achieved with AFLP markers makes it an efficient tool for mapping in shrimp. A total of 429 AFLP markers were identified in the mapping family using $100 \mathrm{EcoRI} / \mathrm{Msel}$ primer combinations, with an average of 8 polymorphic bands per primer pairs. The average number of AFLP polymorphic markers produced from each primer combination was relatively lower than those in $P$. monodon [27], $P$. japonicus [8] and L. vannamei [9], but is almost similar to those generated 7.1 in $P$. chinensis [10], and 9.7 in Portunus trituberculatus [28]. This might be due to the differences among species or the possibility that the $L$. vannamei and $P$. chinensis used have low levels of diversity.

SSR markers have become markers of choice for construction of framework linkage maps due to their wideranging abundance, high polymorphism rate, Mendelian inheritance, and codominant expression [10]. Additionally, because microsatellites are sequence-tagged markers, integration with the linkage maps constructed by other laboratories or physical maps is feasible [29]. In this research, 22 of the 25 SSR markers (88\%) were assigned to the framework map, which was higher than that in $P$. chinensis [10]. The transfer of SSRs will not only be useful for genetic mapping, but will also be critical for comparative mapping and evolution research within the genus Penaeus. With the progress of microsatellites for $L$. vannamei, they will increase the genome coverage, allowing construction of high-resolution linkage maps.

Segregation distortion among DNA markers has been observed to vary according to species and the nature of their mapping populations. Distorted segregation is a common observation in linkage analysis, and the rate of skewed loci in 
Table 2. Informative microsatellite markers in $L$. vannamei a.

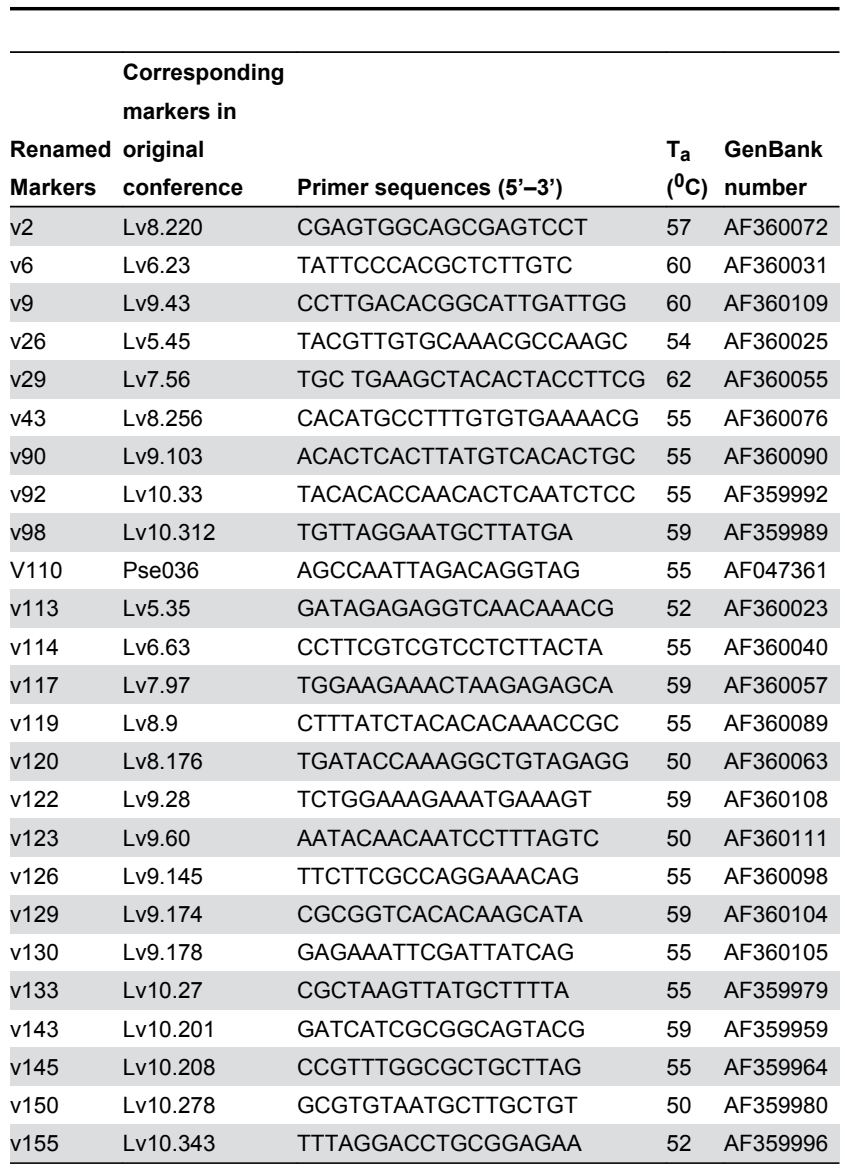

Abbreviations: $T_{a}$ : Annealing temperature ${ }^{a}$ Most of the microsatellite are from Meehan et al. [18], however v110 is from Ball et al. [19].

doi: 10.1371 /journal.pone. $0075206 . t 002$

the $L$. vannamei linkage map $(10 \%)$ is less than $12 \%$ in $P$. chinensis [8] and $16 \%$ of AFLPs in Ictalurus punctatus [11]. However, the percentage is higher than $8 \%$ of AFLPs and microsatellite markers in Oreochromis niloticus [30], and $8 \%$ of AFLPs in C. virginica [31]. The cause of segregation distortion in the linkage map of this research may be associated with the following two factors: (1) presence of genome structure differences between parents of mapping populations [32]; and (2) errors in marker genotyping. Besides these two factors, other research indicates that there is a biased selection of parental genotypes during $F_{2}$ population development. Other reported explanations for the reason include loss of chromosomes [33], presence of gene conversion events [34], and homologous recombination that may cause segregation distortion [35].

\section{Linkage mapping}

In the current map, newly developed SSR markers [10] were mapped in intervals of fragments with AFLPs to decrease the map distance between markers. Compared with the previous map [8], the genetic map presented in this research is improved, with an average genetic distance of $7.6 \mathrm{cM}$ between adjacent markers and a maximum marker interval of $32.8 \mathrm{cM}$ (LG45) (Figure 1). In contrast, Li et al. [8] reported that a total of 30 markers placed on maps with only two markers (m82.7 and m78.5) mapped into the targeted QTL LGs. Marker m82.7 was positioned in the QTL region of LG1 $(103.8 \mathrm{cM})$, while marker m78.5 was in the middle of LG25 (outside of the suggestive QTL region of interest, $44.5 \mathrm{cM}$ ). Although we generally had very good coverage on map, one gap on LG45 was higher than $30 \mathrm{cM}$ in terms of adjacent marker interval (Figure 1). The gap larger than $30 \mathrm{cM}$ (32.8 cM on LG45) could correspond to the recombination of hot regions or marker scale regions. Compared to the average interval of $7.84 \mathrm{cM}$ and 8.30 $\mathrm{CM}$ in the map reported by Li et al. [8], the map presented here is more saturated $(7.6 \mathrm{cM})$. The map provides good coverage of the $L$. vannamei genome $(79.50 \%)$.

LGs (49) obtained in the map developed in this research were higher than the chromosome number $(n=44)$. This genome size is quite large, which may be a result of the many chromosomes of this species and chromosome interferences. The genetic map length should reflect the differences in recombination frequency; nevertheless, the total genetic map length $\left(G_{o a}\right)$ was $3627.6 \mathrm{cM}$ and the estimated genome length $\left(G_{e}\right)$ was $4563.0 \mathrm{cM}$. This phenomenon could be attributed to several factors such as bias in collection of shrimp used for mapping population, number of markers used on map construction, and density and distribution of markers.

\section{QTL analysis}

Genetic linkage maps allow a complete identification and the location of QTL for MAS and hence can be used in programs of genetic improvement in aquaculture. Physical maps enable the integration of linkage maps and karyotypes and are essential tools for comprehensive comparative genomic studies [36]. Moreover, the existence of a well-characterized physical map makes it more feasible to undertake a whole genome sequencing project [37]. In this work we reported the localization of growth-related genes, which showed statistically significant association with growth traits on $L$. vannamei chromosomes. A total of 14 QTLs were identified, including three QTLs for BW, three QTLs for TL, two QTLs for BL, three QTLs for PCL and one QTL for FASD, TASD and FASW.

For BW trait, the most dominant QTL (BW-3) was located on LG25, which explained $18.34 \%$ of phenotypic variation and indicated high additive effect (73.21), implying this locus should be a major genomic position controlling BW trait. For PCL trait, three major QTLs (PCL-1, PCL-2 and PCL-3) explained phenotypic variation ranging from 4.32 to $10.57 \%$ and showed all positive additive effect values, demonstrating that these three loci have great positive effect on PCL. Thus, it can be expected that high BW trait and short PCL trait could be well inherited in progeny if FG shrimp was used as female parent in breeding plans.

Numerous QTLs controlling different traits were clustered in very close interval of the same LG, for example, QTL TL-1 and BW-1 were identified in 16.4 and $45.9 \mathrm{cM}$ interval on LG1; QTL BW-2 and PCL-2 were identified in the same chromosome 

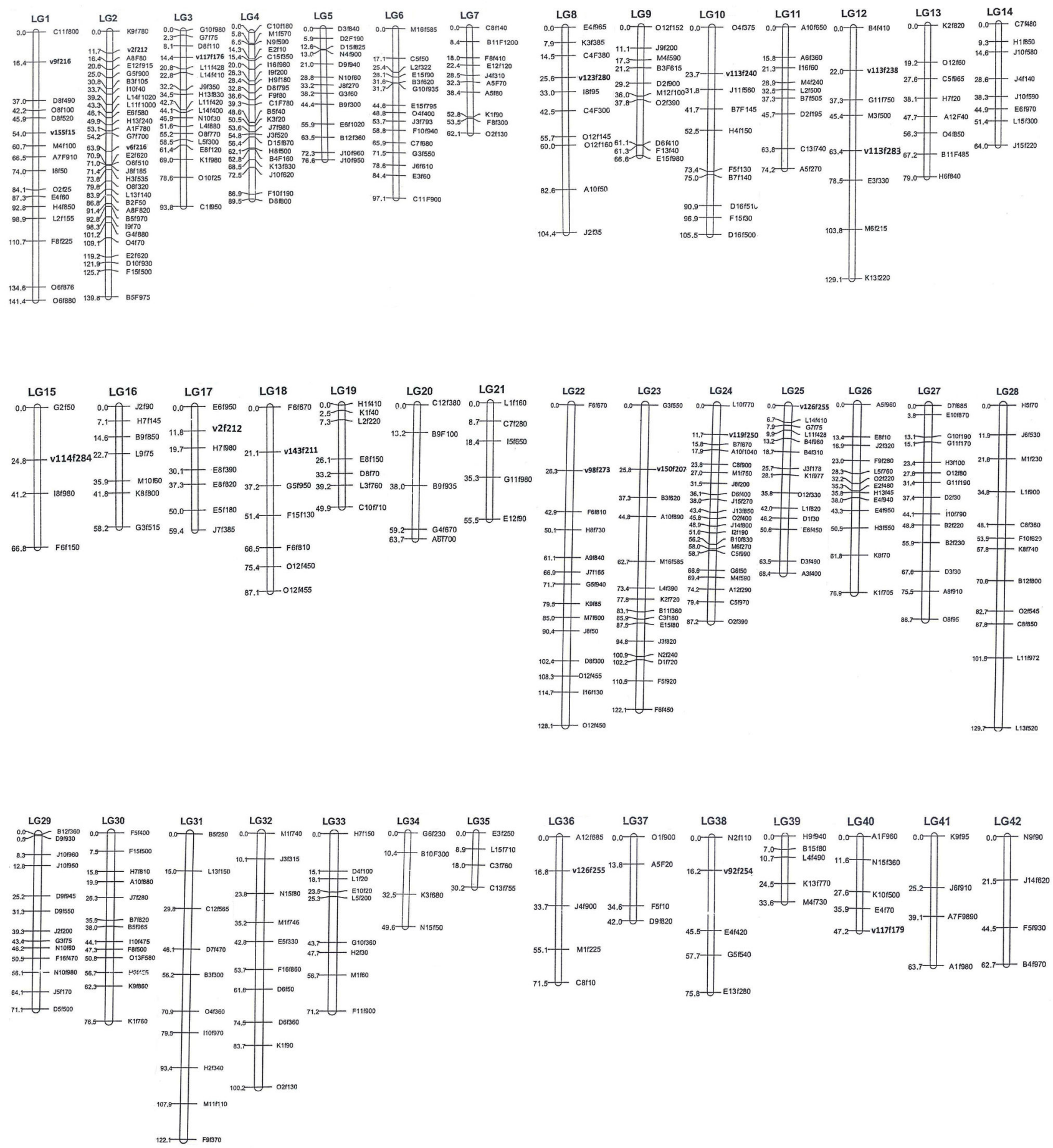

Figure 1. New linkage map in $L$. vannamei based on AFLP and SSR markers. Marker names are shown on the right and the adjacent marker spacing is displayed on the left in Kosambi centimorgans (cM). AFLP markers are in plain typeface and SSR markers are in bold.

doi: 10.1371/journal.pone.0075206.g001

region (44.4 cM) on LG5; QTL FASD-1 and FASW-1 were identified in 16.8 and $33.7 \mathrm{cM}$ interval on LG36. The clustering of QTLs indicated the tight linkage of various genetic positions or the same chromosome region shared by many various QTLs. This tight linkage was confirmed by reported results from quantitative traits analysis [38]. Identification of QTLs 
Table 3. Summary statistics of genetic linkage maps of $L$. vannamei.

\begin{tabular}{|c|c|}
\hline & Values (distored markers) \\
\hline Segregating markers & $513(47)$ \\
\hline Number of markers in linkage analysis & $505(38)$ \\
\hline \multicolumn{2}{|l|}{ Mapped markers } \\
\hline AFLPs & $429(33)$ \\
\hline SSRs & $22(1)$ \\
\hline Unlinked doublets & 6 \\
\hline Unlinked single markers & $43(4)$ \\
\hline Linkage groups & 49 \\
\hline Average number of markers per group & 8 \\
\hline Minimum number of markers per group & 3 \\
\hline Maximum number of markers per group & 28 \\
\hline Average marker spacing (cM) & 7.6 \\
\hline Maximum marker spacing(cM) & 32.8 \\
\hline Minimum length of linkage group(cM) & 13.1 \\
\hline Maximum length of linkage group(cM) & 141.4 \\
\hline \multicolumn{2}{|l|}{ Observed genome length(cM) } \\
\hline $\mathrm{G}_{\text {of }}$ & 3313.9 \\
\hline $\mathrm{G}_{\mathrm{oa}}$ & 3627.6 \\
\hline \multicolumn{2}{|l|}{ Estimated genome length (cM) } \\
\hline $\mathrm{G}_{\mathrm{e} 1}$ & 4543.0 \\
\hline $\mathrm{G}_{\mathrm{e} 2}$ & 4583.0 \\
\hline $\mathrm{G}_{\mathrm{e}}$ & 4563.0 \\
\hline \multicolumn{2}{|l|}{ Genome coverage (\%) } \\
\hline $\mathrm{C}_{\text {of }}$ & 72.63 \\
\hline $\mathrm{C}_{\mathrm{oa}}$ & 79.50 \\
\hline
\end{tabular}

Abbreviations: $G_{o f}$ : length of the framework map; $G_{o a}$ : total length considering all markers; $\mathrm{G}_{\mathrm{e}}$ : the estimated genome length that is calculated by the average of the two estimates $\left(G_{e 1}\right.$ and $\left.G_{e 2}\right)$. $C_{o f}$ and $C_{o a}$ : observed genome coverages, determined by $\mathrm{G}_{\mathrm{of}} / \mathrm{G}_{\mathrm{e}}$ and $\mathrm{G}_{\mathrm{oa}} / \mathrm{G}_{\mathrm{e}}$

doi: 10.1371/journal.pone.0075206.t003

influencing many traits could increase the efficiency of MAS and enhance genetic progress [39]. Meanwhile, the corresponding clustering of numerous QTLs affecting numerous traits provides an explanation for positive correlation among various traits.

\section{Comparative mapping}

Comparative mapping between $L$. vannamei and $P$. japonicus can give us new insights into the evolution of shrimp species. In this paper, locus $\mathrm{H} 13 \mathrm{f} 380$ was mapped on LG3 and locus J3f178 was mapped on LG25, however, they were both conserved with the same chromosome 25 of $P$. japonicus. It is speculated that LG3 and LG25 probably correspond to a single orthologous chromosome in $P$. japonicus.

Interestingly, locus D8f520 linked with a QTL affecting BW and PCL traits was conserved with a growth hormone gene of $P$. monodon (GenBank accession number: G0075401). This provides a new insight into the genetic base of QTL affecting BW and PCL.

In conclusion, the present map attains two goals: (1) the intermarker distance on this framework map and the good coverage provides enough marker density for mapping of quantitative traits; and (2) the map provides an effective tool for genetic analysis and manipulations. However, this map needs further improvement and QTLs also needs further precise location, but it will still be a very useful tool for shrimp genetic research and selective breeding schemes in the future. Combining next generation sequencing and genotyping technological advances, more markers could be added to genetic map of shrimp to improve the genetic map quality. Further research of growth-related traits will help us expand our knowledge of crustacean growth and to produce high-quality shrimp products. 

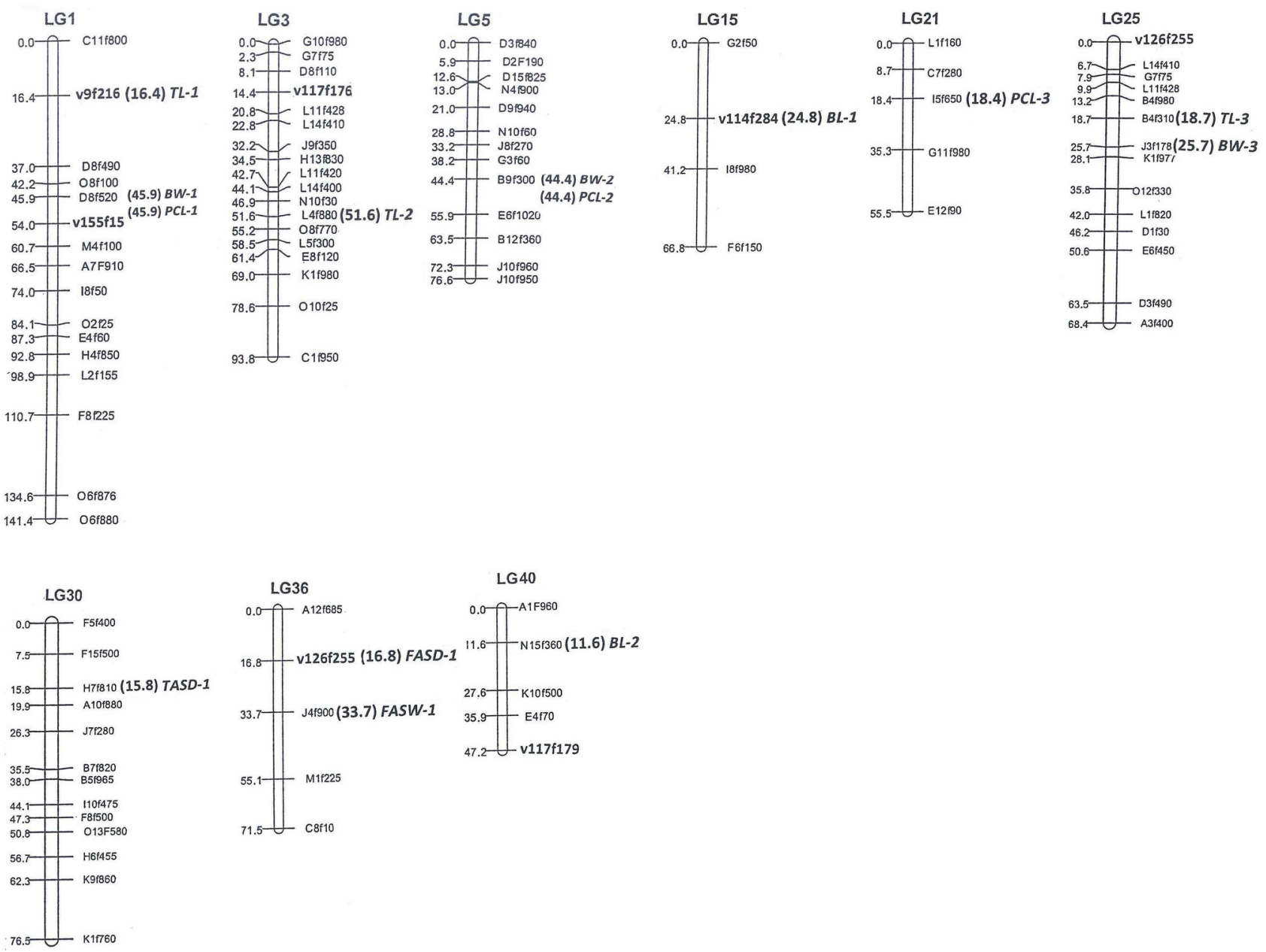

Figure 2. Putative QTLs for growth-related traits in L. vannamei. QTLs, Quantitative trait loci.

doi: 10.1371/journal.pone.0075206.g002 
Table 4. Putative QTLs and their genetic effects for growth-related traits in L. vannamei.

\begin{tabular}{|c|c|c|c|c|c|c|c|}
\hline Traits & QTL & Linkage groups & Markers & Position & $L O D$ value & Additive effect & Variance (\%) \\
\hline \multirow[t]{3}{*}{ BW } & BW-1 & 1 & D8f520 & 45.90 & 3.79 & 71.12 & 15.86 \\
\hline & BW-2 & 5 & B9f300 & 44.40 & 3.51 & 29.10 & 2.62 \\
\hline & BW-3 & 25 & $J 3 f 178$ & 25.70 & 4.44 & 73.21 & 18.34 \\
\hline \multirow[t]{3}{*}{ TL } & TL-1 & 1 & v9f216 & 16.40 & 3.44 & 1.13 & 14.74 \\
\hline & TL-2 & 3 & L4f880 & 51.60 & 4.16 & 2.12 & 10.96 \\
\hline & TL-3 & 25 & $\mathrm{~B} 4 \mathrm{f} 310$ & 18.70 & 3.30 & 1.80 & 13.93 \\
\hline \multirow[t]{2}{*}{ BL } & BL-1 & 15 & v114f284 & 24.80 & 3.99 & 2.80 & 45.95 \\
\hline & BL-2 & 40 & $\mathrm{~N} 15 f 360$ & 11.60 & 4.18 & 0.44 & 42.78 \\
\hline FASD & FASD-1 & 36 & v126f255 & 16.80 & 4.69 & 0.50 & 61.42 \\
\hline TASD & TASD-1 & 30 & H7f810 & 15.80 & 4.13 & 0.50 & 56.10 \\
\hline FASW & FASW-1 & 36 & J4f900 & 33.70 & 4.23 & 0.50 & 56.20 \\
\hline \multirow[t]{3}{*}{ PCL } & PCL-1 & 1 & D8f520 & 45.90 & 6.65 & 0.29 & 7.62 \\
\hline & PCL-2 & 5 & B9f300 & 44.40 & 6.34 & 0.34 & 4.32 \\
\hline & PCL-3 & 21 & $15 f 650$ & 18.40 & 6.98 & 0.41 & 10.57 \\
\hline
\end{tabular}

doi: 10.1371 /journal.pone.0075206.t004

Table 5. Comparative chromosome of AFLP markers in linkage map of $L$. vannamei and $P$. japonicus.

\begin{tabular}{lll}
\hline AFLP locus & Linkage group in L. vannamei & Chromosome in $P$. japonicus \\
\hline D8f520 & LG1 & Chrom1 \\
M4f100 & LG1 & Chrom17 \\
H13f380 & LG3 & Chrom25 \\
J3f178 & LG25 & Chrom25 \\
I8f980 & LG15 & Chrom9 \\
E8f150 & LG19 & Chrom11 \\
L13f150 & LG31 & Chrom23 \\
C13f755 & LG35 & Chrom19 \\
\hline
\end{tabular}

doi: 10.1371/journal.pone.0075206.t005 


\section{Acknowledgements}

We wish to thank the staff of the hatchery and growout at Hainan Guangtai Ocean Breeding Company Limited (Wenchang Breeding Base) in Hainan province, China, for active participation in the production of the experimental shrimp.

\section{References}

1. Andriantahina F, Liu X, Feng T, Xiang J (2013a) Current status of genetics and genomics of reared penaeid shrimp: information relevant to access and benefit sharing. Mar Biotechnol 15: 399-412. doi: 10.1007/s10126-013-9500-9. PubMed: 23529408.

2. Mackay TFC, Stone EA, Ayroles JF (2009) The genetics of quantitative traits: challenges and prospects. Nat Rev Genet 10: 565-577. doi: 10.1038/ni0609-565. PubMed: 19584810.

3. Poormohammad Kiani S, Maury P, Nouri L, Ykhlef N, Grieu P et al. (2009) QTL analysis of yield-related traits in sunflower under different water treatments. Plants Breed 128: 363-373. doi:10.1111/j. 1439-0523.2009.01628.x.

4. Andriantahina F, Liu X, Huang H, Xiang J, Yang C (2012b) Comparison of reproductive performance and offspring quality of domesticated Pacific white shrimp, Litopenaeus vannamei. Aquaculture 324-325: 194-200. doi:10.1016/j.aquaculture.2011.10.026.

5. Andriantahina F, Liu X, Huang H, Xiang J (2012a) Response to selection, heritability and genetic correlations between body weight and body size in Pacific white shrimp, Litopenaeus vannamei. Chin J Oceanol Limnol 30: 200-205. doi:10.1007/s00343-012-1066-2.

6. Andriantahina F, Liu X, Huang H, Xiang J (2013b) Selection for Growth Performance of Tank Reared Pacific white shrimp, Litopenaeus vannamei. Chin J Oceanol Limnol 31: 534-541. doi:10.1007/ s00343-013-2220-1.

7. Maneeruttanarungroj C, Pongsomboon S, Wuthisuthimethavee $\mathrm{S}$, Klinbunga S, Wilson KJ et al. (2006) Development of polymorphic expressed sequence tag derived microsatellites for the extension of the genetic linkage map of the black tiger shrimp (Penaeus monodon). Anim Genet 37: 363-368. doi:10.1111/j.1365-2052.2006.01493.x. PubMed: 16879347.

8. Li Y, Dierens L, Byrne K, Miggiano E, Lehnert S et al. (2006) QTL detection of production traits for the Kuruma prawn Penaeus japonicus (Bate) using AFLP markers. Aquaculture 258: 198-210. doi:10.1016/ j.aquaculture.2006.04.027.

9. Zhang L, Yang C, Zhang T, Li L, Zhang XM et al. (2007) A genetic linkage map of Pacific white shrimp (Litopenaeus vannamei): sex-linked microsatellite markers and high recombination rates. Genetica 131: $37-$ 49. doi:10.1007/s10709-006-9111-8. PubMed: 17043742.

10. Liu B, Wang Q, Li J, Liu P, He YY (2010) A genetic linkage map of marine shrimp Penaeus (Fenneropenaeus) chinensis based on AFLP, SSR, and RAPD markers. Chin J Oceanol Limnol 28: 815-825. doi: 10.1007/s00343-010-9915-3.

11. Liu Z, Karsi A, Li P, Cao D, Dunham R (2003) An AFLP-based genetic linkage map of channel catfish (Ictalurus punctatus) constructed by using an interspecific hybrid resource family. Genetics 165: 687-694. PubMed: 14573480.

12. Sekino M, Hara M (2007) Linkage Maps for the Pacific Abalone (Genus Haliotis) Based on Microsatellite DNA Markers. Genetics 175: 945-958. doi:10.1534/genetics.106.065839. PubMed: 17151239 .

13. Wang SC, Basten J, Zeng ZB (2007) Windows QTL Cartographer 2.5. Raleigh, NC: Department of Statistics, North Carolina State University. Available: http://statgen.ncsu.edu/qtlcart/WQTLCart.htm. Accessed 25 April 2013.

14. Xia JH, Liu F, Zhu ZY, Fu J, Feng J et al. (2010) A consensus linkage map of the grass carp (Ctenopharyngodon idella) based on microsatellites and SNPs. BMC Genomics 11: 135. doi: 10.1186/1471-2164-11-135. PubMed: 20181260.

15. Sambrook J, Russell DW (2001) Molecular Cloning: A Laboratory Manual. Cold Spring Harbor Laboratory Press. 2344pp.

16. Vos P, Hogers R, Bleeker M, Reijans M, van de Lee T et al. (1995) AFLP, a new technique for DNA fingerprinting. Nucleic Acids Res 23: 4407-4414. doi:10.1093/nar/23.21.4407. PubMed: 7501463.

17. Li L, Xiang J, Liu X, Zhang Y, Dong B et al. (2005) Construction of AFLP-based genetic linkage map for Zhikong scallop, Chlamys farreri Jones et Preston and mapping of sex-linked markers. Aquaculture 245: 63-73. doi:10.1016/j.aquaculture.2004.12.015.

\section{Author Contributions}

Conceived and designed the experiments: FA XL. Performed the experiments: $F A X L ~ H H$. Analyzed the data: $F A X L$. Contributed reagents/materials/analysis tools: $F A \quad X L H H$. Wrote the manuscript: FA XL.

18. Meehan D, Xu Z, Zuniga G, Alcivar-Warren A (2003) High frequency and large number of polymorphic microsatellites in cultured shrimp, Penaeus (Litopenaeus) vannamei [Crustacea: Decapoda]. Mar Biotechnol 5: 311-330. doi:10.1007/s10126-002-0092-z. PubMed: 14719161.

19. Ball AO, Leonard SRW (1998) Chapman, Characterization of (GT)n microsatellites from native white shrimp (Penaeus setiferus). Mol Ecol 7: 1251-1253. PubMed: 9734081.

20. Manly KF, Cudmore JRH, Meer JM (2001) Map Manager QTX, crossplatform software for genetic mapping. Mamm Genome 12: 930-932. doi:10.1007/s00335-001-1016-3. PubMed: 11707780.

21. Voorrips RE (2002) MapChart: Software for the Graphical Presentation of Linkage Maps and QTLs. J Hered 93: 77-78. doi:10.1093/jhered/ 93.1.77. PubMed: 12011185.

22. Chakravarti A, Lasher LK, Reefer JE (1991) A maximum likelihood method for estimating genome length using genetic linkage data. Genetics 128: 175-182. PubMed: 2060775.

23. Zhang TS, Liu P, Li J, Kong J, Wang QY (2006) Preliminary study on specific microsatellites markers related to growth trait in Fenneropenaeus chinensis. Mar. Fish Res 5: 34-38.

24. Zeng ZB (1994) Precision mapping of quantitative trait loci. Genetics 136: 1457-1468. PubMed: 8013918.

25. Du ZQ, Ciobanu DC, Onteru SK, Gorbach D, Mileham AJ et al. (2010) A gene-based SNP linkage map for pacific white shrimp, Litopenaeus vannamei. Anim Genet 41: 286-294. doi:10.1111/j. 1365-2052.2009.02002.x. PubMed: 19968647.

26. Woram RA, Gharbi K, Sakamoto T, Hoyheim B, Holm LE et al. (2003) Comparative Genome Analysis of the Primary Sex Determining Locus in Salmonid Fishes. Genome Res 13: 272-280. doi:10.1101/gr.578503. PubMed: 12566405.

27. Wilson K, Li YT, Whan V, Lehnert SA, Byrne K et al. (2002) Genetic mapping of the black tiger shrimp Penaeus monodon with amplified fragment length polymorphism. Aquaculture 204: 297-309. doi: 10.1016/S0044-8486(01)00842-0.

28. Luo Y, Gao BQ, Liu P, Li J, Dai FY (2010) Construction of genetic linkage map of Portunus trituberculatus. Prog. Fish Sci 31: 56-65.

29. Liu P, Li J, He YY, Kong J, Wang Q (2004) Present situation and protective measures of genetic resources in Fenneropenaeus chinensis. Mar. Fish Res 25: 80-85.

30. Kocher TD, Lee WJ, Sobolewska H, Penman D, McAndrew B (1998) A genetic linkage map of a cichlid fish, the tilapia (Oreochromis niloticus). Genetics 148: 1225-1232. PubMed: 9539437.

31. Yu Z, Guo X (2003) Genetic linkage map of the eastern oyster Crassostrea virginica gmelin. Biol Bull 204: 327-338. doi: 10.2307/1543603. PubMed: 12807709.

32. Hwang T-Y, Sayama T, Takahashi M, Takada $Y$, Nakamoto $Y$ et al. (2009) High-density integrated linkage map based on SSR markers in soyabean. DNA Res 16: 213-225. doi:10.1093/dnares/dsp010. PubMed: 19531560.

33. Kasha KJ, Kao KN (1970) High frequency haploid production in barley (Hordeum vulgare L.). Nature 225: 874-876. doi:10.1038/225874a0. PubMed: 16056782.

34. Nag DK, White MA, Petes TD (1989) Palindromic sequences in heteroduplex DNA inhibit mismatch repair in yeast. Nature 340: 318320. doi:10.1038/340318a0. PubMed: 2546083.

35. Armstrong KC, Keller WA (1982) Chromosome pairing in haploids of Brassica oleracea. Can J Genet Cytol 24: 735-739.

36. Sánchez-Ramos I, Cross I, Mácha J, Martínez-Rodríguez G, Krylov V et al. (2012) Assessment of Tools for Marker-Assisted Selection in a Marine Commercial Species: Significant Association between MSTN-1 Gene Polymorphism and Growth Traits. Sci. World J. doi: 10.1100/2012/369802.

37. Ng SHS, Artieri CG, Bosdet IE (2005) A physical map of the genome of Atlantic salmon, Salmo salar. Genomics 86: 396-404. doi:10.1016/ j.ygeno.2005.06.001. PubMed: 16026963. 
38. Wang J, He A, Ma Y, Wang C (2012) Genetic map construction and quantitative trait locus (QTL) analysis on growth-related traits in common carp (Cyprinus carpio L.). Afr J Biotechnol 11: 7874-7884.

39. Upadyayula N, da Silva HS, Bohn MO, Rocheford TR (2006) Genetic and QTL analysis of maize tassel and ear inflorescence architecture.
Theor Appl Genet 112: 592-606. doi:10.1007/s00122-005-0133-x. PubMed: 16395569 